\title{
Generation of Steiner Quadruple Systems
}

\author{
H. M. I. C. Herath ${ }^{1}$, A. A. I. Perera ${ }^{2}$, A. A. C. A. Jayathilake ${ }^{3}$ \\ ${ }^{1,2,3}$ Department of Mathematics, Faculty of Science, University of Peradeniya
}

\begin{abstract}
A block design with $v$ points and a set of blocks $\mathcal{B}$ where each block is a b-subset of $v$, such that each point is contained in exactly $r$-blocks \& each distinct $k$ point is contained in exactly $\lambda$-blocks, known as a $t-(v, k, \lambda)$-design which plays an important role in design theory. A Steiner system is a special type of $t-(v, k, \lambda)$ design with $\lambda=1 \& k=t+1$. Among these steiner systems, steiner quadruple systems (SQS) and Steiner Triple Systems(STS) are the designs that are widely used in constructing designs. In this work, we present an effective automated method of finding SQS design of $2^{n}$ vertices, where $n \in \mathbb{Z}$, with the help of STS. We begin with a set of blocks of a known STS, and the binary representation of all those blocks was constructed. Then, a MATLAB program was used to find the blocks of a SQS which related to the SQS that we have chosen. The next step was to find the corresponding incidence matrix for the design obtained in the first step and another separate program was designed to obtain the incidence matrix. Finally, with the help of this incidence matrix, a new program was implemented to obtain a complete graph which corresponds to the SQS obtained above. These blocks have several properties such that triply transitive, automorphism-free, heterogeneous for $n \geq 3$, resolvable \& non-disjoint. By extending the program for Steiner triple systems blocks of $S T S\left(2^{n}-1\right)$-design number of blocks, incidence matrices, and complete graphs with $2^{n}-1$ number of vertices were obtained as another result. These Steiner quadruple systems and Steiner triple systems can be used in fields of communication, cryptography, and networking.
\end{abstract}

Keywords: Block designs, Steiner quadruple system, Steiner triple system

\section{Introduction}

A block design is a design with $v$ points and a set of blocks $\mathcal{B}$ where each block contains subsets with $b$ points called $b$ subset, such that each point is contained in exactly $r$-blocks \&any pair of points is contained in exactly $\lambda$-blocks, where $r, \lambda$ are constants.

A $\mathrm{t}-(\mathrm{v}, \mathrm{k}, \lambda)$-design is a design with parameters $\mathrm{v}, \mathrm{k}, \lambda$ where $(\mathrm{X}, \mathcal{B})$ is a pair with $v$-point set \& the collection of $\mathrm{k}$ subsets of $\mathrm{X}$, called blocks with the property that any $\mathrm{t}$ points are contained in exactly $\lambda$ blocks $\& v>k \geq \lambda$

A $t-(v, k, \lambda)$ design with $\lambda=1 \& k=t+1$ is called a Steiner System which was first introduced by the Swiss Mathematician Jacob Steiner(Anderson \& Honkala, 1997).

Steiner quadruple system is a pair $(\mathrm{X}, \mathcal{B})$ where $X$ is a finite set and $B$ is a collection of 4 -subsets (called quadruple or blocks with 4 elements in each) such that every 3 -subsets of $X$ is contained in exactly one quadruple of $\mathcal{B}$.In other words, a Steiner quadruple system is a Steiner system with $t=$ $3 \& k=4$.It has been shown that $v \equiv 2 \operatorname{or} 4(\bmod 6)$ is the necessary condition for the existence of Steiner quadruple system. (Linder \& Rosa, 1977, p. 148)

There are various types of Steiner quadruple systems. Among them, cyclic SQS, transitive SQS, automorphismfree SQS, heterogeneous SQS, resolvable SQS \& disjoint SQS are some prominent types of SQS(Linder \& Rosa, 1977, pp. 154-173).

A SQS $(v)$ is cyclic if it admits an automorphism $C$ consisting of single cycle of length $v$, where $C$ is called a cyclic automorphism. Also, $v \equiv 2$ or $10(\bmod 24)$ can be identified as the necessary condition for theexistence of cyclic SQS.Further, it is important to note that, there are no cyclic SQS with order $8,14 \& 16$. The next order of thecyclic SQS is 20 and the following figure shows the 15 base quadruples
(Linder \& Rosa, 1977, pp. 155-157).

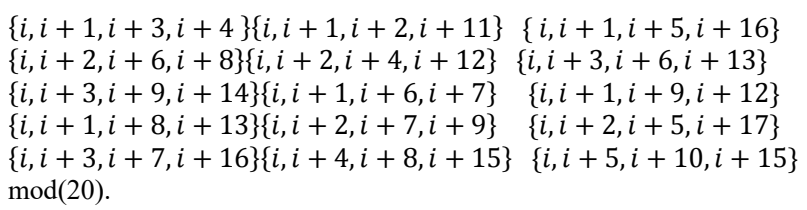
$\bmod (20)$

If $(X, \mathcal{B})$ is a cyclic $S Q S$, then the quadruples of $\mathcal{B}$ can bepartitioned into orbits under the action of the cyclic group generated by $C$. Each orbit of quadruples is completely determined by any one of its quadruples, and the set $\mathcal{B}$ is determined by a collection of quadruple called base quadruples containing one quadruple from each orbit. (Linder \& Rosa, 1977, pp. 154-155)

SQS whose automorphism group acts transitively on the elements is called transitive SQS(Linder \& Rosa, 1977, p. 157). Cyclic Steiner quadruple systems are transitive. Any three points in affine geometryAG(n, 2) determines a plane of $\operatorname{AG}(n, 2)$ and this plane contains just one more point of AG(n, 2) (Linder \& Rosa, 1977, p. 157). So in $\operatorname{SQS}\left(2^{n}\right)$ design, any three points in a block is uniquely determined the fourth according to the affine geometry. Therefore, the automorphism group of $\mathrm{SQS}\left(2^{\mathrm{n}}\right)$ design is triply transitive.

Automorphism group of $\operatorname{STS}\left(2^{\mathrm{n}}\right)$ which is isomorphic to the design of points and lines of affine space $A G(n, 3)$ over GF(3) is also triply transitive. Using that design, sharply triply transitive SQS can be constructed. Another interesting class of transitive SQS (flag-transitiveSQS) can be constructed using STS with prime power order $v \equiv 7(\bmod$ 12).

A SQS is automorphism-free, if it admits no non trivialautomorphism. If Steiner quadruplesystem is an automorphism-free then $v \geq 16$. This is an example of automorphism-free SQS(16)(Linder \& Rosa, 1977, p. 159). 


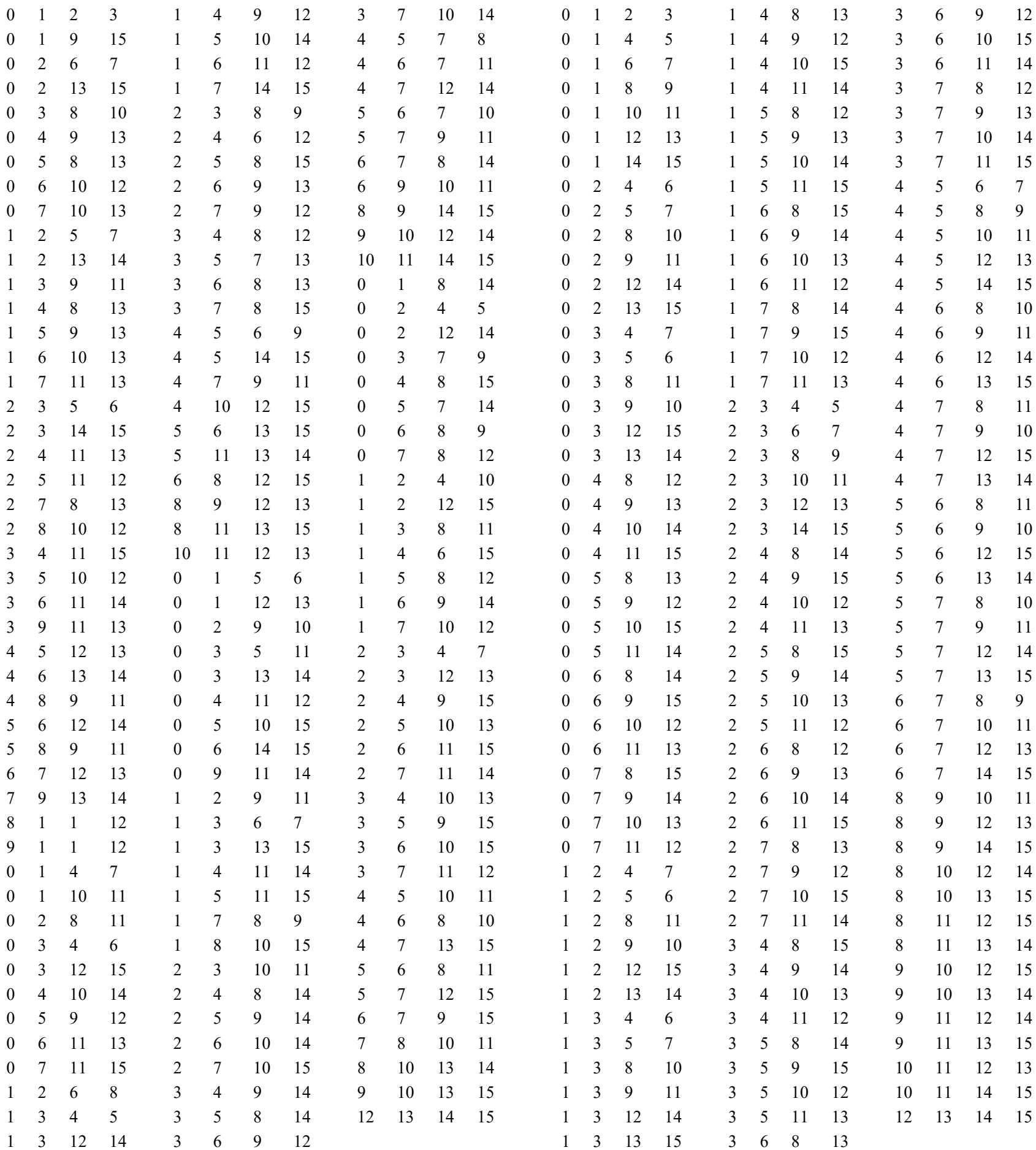

A SQS $(X, \mathcal{B})$ with number of non-isomorphic derived STS $\left(\mathrm{X}_{y}, \mathcal{B}(\mathrm{y})\right)$ of $(\mathrm{X}, \mathcal{B})$ equals to the order of SQSis called Heterogeneous. If there exists a heterogeneous $\mathrm{SQS}(v)$, then there exists a heterogeneous $\operatorname{SQS}(2 v)$. As a consequence, a heterogeneous $\mathrm{SQS}(v)$ exists for every $v$ of the form $v=2^{k+3}$ or $v=10.2^{k}$ where $k$ is a positive integer. And also there is a conjecture that a heterogeneous SQS exists if and only if $v \geq 16$. It has been found that $\operatorname{SQS}(16)$ \& SQS(20) are hererogeneous. A heterogeneous SQS is necessarily automorphism-free and converse is not true. SQS(16) is a example for that(Linder \& Rosa, 1977, pp. 170171).
A Steiner quadruple system $(\mathrm{X}, \mathcal{B})$ is said to be resolvable if its set of quadruples $\mathcal{B}$ can be partitioned into parallel classes. A necessary condition for the existence of a resolvable SQS is $v \equiv 4$ or $8(\bmod 12)$ as $v$ must be divisible by four. The unique SQS(8) is an example of a resolvable SQS(Hartman, 1987).

For any $\operatorname{SQS}(v)=\mathrm{S}(3,4, v)$ can be partitioned into $B_{1}, B_{2}, B_{3}, \ldots \ldots \ldots B_{v-2 / 2}$ such that $\left(X, B_{i}\right)$ is an $\mathrm{S}(2,4, v)$ for each $i=1,2,3, \ldots \ldots \ldots,(v-2) / 2$, then $\operatorname{SQS}(v)$ is 2resolvable. If $B_{i}$ can be partitioned in to paralleled classes, then $\operatorname{SQS}(v)$ is called doubly resolvable. Then the necessary condition for $\operatorname{SQS}(v)$ to be doubly resolvable is $v \equiv 4(\bmod$ 12) and it provides that $\mathrm{SQS}(16)$ is either 2-resolvable or doubly resolvable. A 1 -resolvable $\operatorname{SQS}(v)$ need not be 2- 


\section{International Journal of Science and Research (IJSR) \\ ISSN (Online): 2319-7064}

Index Copernicus Value (2013): 6.14 | Impact Factor (2014): 5.611

resolvable.

Two Steiner quadruple system $\left(\mathrm{X}, \mathcal{B}_{1}\right) \&\left(X, \mathcal{B}_{2}\right)$ are disjoint if $\mathcal{B}_{1} \cap \mathcal{B}_{2}$ is empty. If $d(v)$ be the maximum number of pair wise disjoint SQS of order $v$, then $d(v) \geq v-3$ for every $v \geq 4$.If $D(v)$ be the maximum number of pair wise disjoint STS of order $v$, then obviously $d(v) \leq D(v-1)$.

Triples can be packed and covered by quadruples. There are two methods knownas, minimal covering quadruple system and maximal packing quadruple system.

A minimal covering quadruple system is a pair $(\mathrm{S}, \mathcal{B})$ where $\mathrm{S}$ is an $\mathrm{n}$-set and $\mathcal{B}$ is a minimal collection of quadruples of $\mathrm{S}$ such that every 3 -subset of $S$ is contained in at least one quadruple of $\mathcal{B}$ and it is denoted by $C(3,4, n)$. (Linder \& Rosa, 1977, pp. 176-177)

Also, a maximal packing quadruple system is a pair $(\mathrm{Q}, \mathcal{B})$ where $\mathcal{B}$ is a maximal collection of quadruples of $Q$ such that every 3 -subset of $Q$ is contained in at most one quadruple of $\mathcal{B}$ and denoted by $P(3,4, n)$. (Linder \& Rosa, 1977, pp. 176177)

Further, notice that $|P(3,4, n)| \leq l(n) \&|C(3,4, n)| \geq L(n)$ where

$$
\begin{aligned}
& l(n)=\left[\frac{1}{4} n\left[\frac{1}{3}(n-1)\left[\frac{1}{2}(n-2)\right]\right]\right] \& \\
& L(n)=\left[\frac{1}{4} n\left[\frac{1}{3}(n-1)\left[\frac{1}{2}(n-2)\right]\right] .\right.
\end{aligned}
$$

Here $\lfloor x\rfloor$ denotes the largest integer not exceeding $x$ and $\lceil x\rceil$ denote the smallest integer not less than $x$.

In this work, our ultimate goal is to find the Steiner Quadruple Systems of higher orders using an automated method developed using MATAB. The next section illustrates the steps of the method.

\section{Methodology}

Steps to obtain the SQS (2n)-design are discussed in the following context. As the first step a known SQS, 3(8,4,1)design has been considered thi design is the $\operatorname{SQS}\left(2^{3}\right)$ and it can be geometrically represented by using a cube.

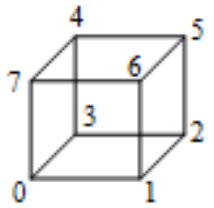

There are total number of 14 blocks $\&$ those are $\{0123,0145,0167,0246,0257,0347,0356$, $1247,1256,1346,1357,2345,2367,4567\}$

These blocks were written in binary numbers \& there column wise sum was obtained as follows separately. (Table 1).
Table 1

\begin{tabular}{|c|c|c|}
\hline Block & Binary representation & Column wise sum \\
\hline 0123 & $000,001,010,011$ & $0,2,2$ \\
\hline 0145 & $000,001,100,101$ & $2,0,2$ \\
\hline 0167 & $000,001,110,111$ & $2,2,2$ \\
\hline 0246 & $000,010,100,110$ & $2,2,0$ \\
\hline 0257 & $000,010,101,111$ & $2,2,2$ \\
\hline 0347 & $000,011,100,111$ & $2,2,2$ \\
\hline 0356 & $000,011,101,110$ & $2,2,2$ \\
\hline 1247 & $001,010,100,111$ & $2,2,2$ \\
\hline 1256 & $001,010,101,110$ & $2,2,2$ \\
\hline 1346 & $001,011,100,110$ & $2,2,2$ \\
\hline 1357 & $001,011,101,111$ & $2,2,4$ \\
\hline 2345 & $010,011,100,101$ & $2,2,2$ \\
\hline 2367 & $010,011,110,111$ & $2,4,2$ \\
\hline 4567 & $100,101,110,111$ & $4,2,2$ \\
\hline
\end{tabular}

According to the table 1, all separately column wise sum is even $\&$ this is a special characteristic of blocks of $\operatorname{SQS}\left(2^{\mathrm{n}}\right)$ designs. Under that concept a MATLAB program was written to obtain blocks of $\operatorname{SQS}\left(2^{\mathrm{n}}\right)$ design, number of blocks, incidence matrix of those blocks \& complete graph with $2^{\mathrm{n}}$ number of vertices. This program has separated in three sections.

\section{Program 1 (TO FIND BLOCKS OF SQS $\left(2^{\mathrm{n}}\right)$ DESIGN)}

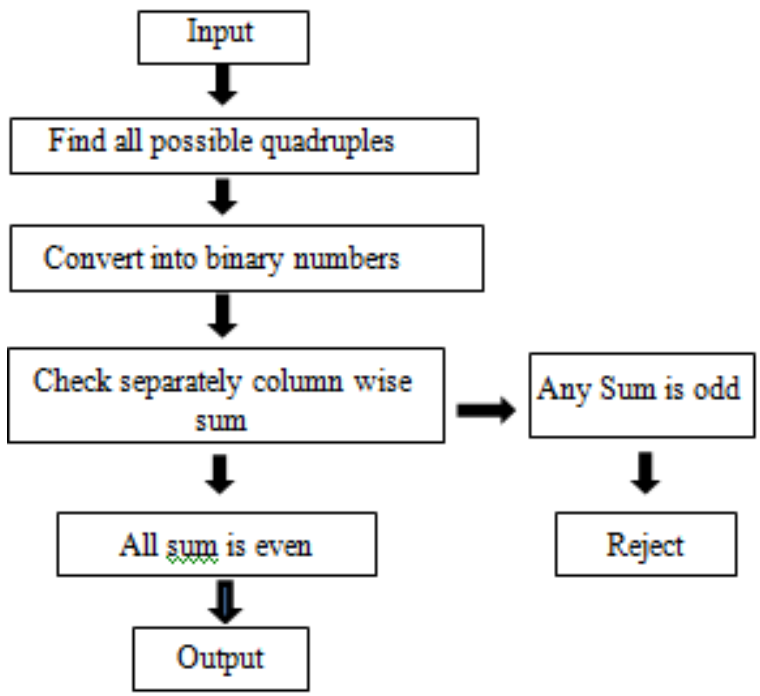

If $v$ be the number of points. Then there are $\left(\begin{array}{l}v \\ 4\end{array}\right)$ number of quadruples. First those quadruples was found. Then converted into binary numbers and separated into sub matrices of order 4.After that column wise sum was taken separately .If separately column wise sum was even, then those blocks were taken as output. If any column wise sum was odd, then those blocks were rejected.

\section{PROGRAM 2 (TO FIND INCIDENCE MATRIX)}


International Journal of Science and Research (IJSR)

ISSN (Online): 2319-7064

Index Copernicus Value (2013): 6.14 | Impact Factor (2014): 5.611

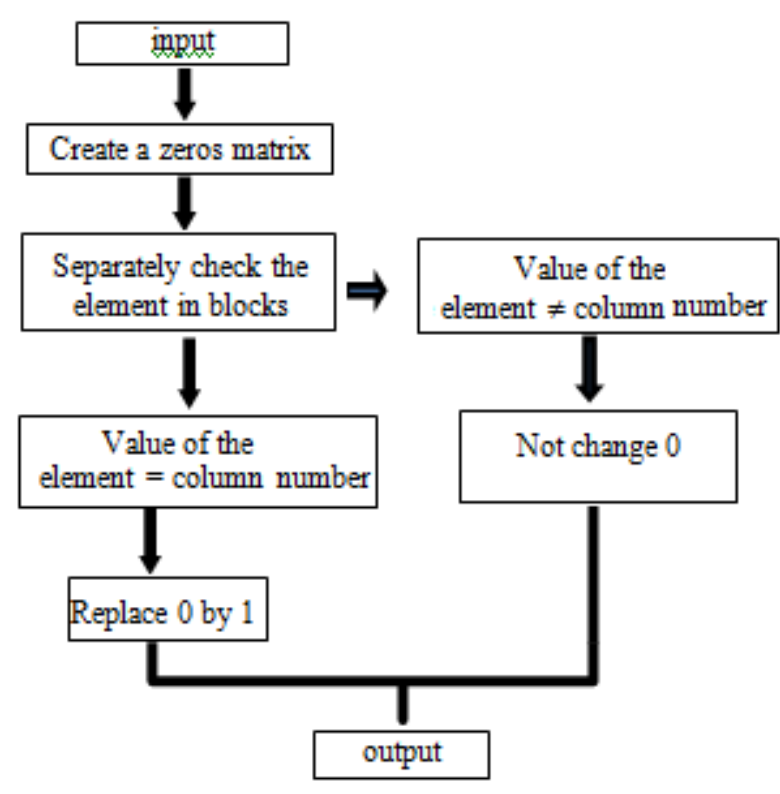

Here input was the previous program output. (i.e , blocks of SQS $2^{\text {n }}$ design). Let $b$ be the total number of blocks $\&$ let $v$ be the number of point. Then zeros $(b, v)$ matrix was created. Elements of each block were checked separately and if in $i^{\text {th }}$ block value of elements are equal to column numbers then in the existence matrix ( $i$,value of the element $)^{\text {th }} 0$ was replaced by 1 for $i=1,2, \ldots \ldots \ldots \ldots \ldots$.If in $i^{\text {th }}$ block, value of elements are not equal to column numbers, then 0 was not changed. Ten incidence matrix was taken as output.

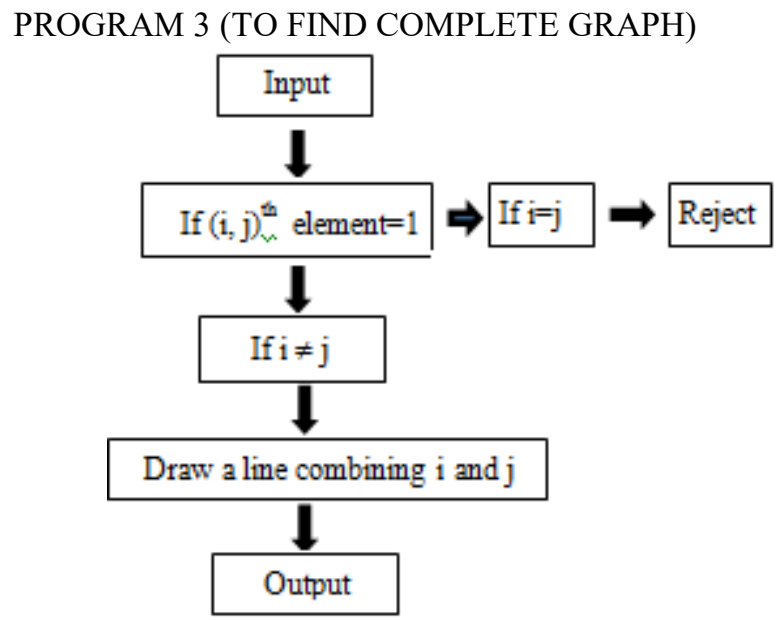

Here also input was previous program output (i.e incidence matrix). Let $i$ be a row $\& j$ be a column. If $(i, j)^{\text {th }}$ element was equal 1 , then checked whether $i=j$ or not for $i=b \& j=$ $v$.If $i=j$, then it was rejected.If $i \neq j$, a line was drawn by joining $i \& j$.A complete graph was taken as output.

\section{METHOD TO FIND STS( $\left.2^{n}-1\right)$-DESIGN}

STS can be taken by overlapping two points of SQS. As an example consider $\mathrm{SQS}\left(2^{3}\right)$-design. Among the blocks of SQS $\left(2^{3}\right)$-design, select blocks which begins the element 0 . Those are, $0123,0145,016$ 7, 0246,025 7,0 34 7, 0 356

By removing zeros from those blocks STS $\left(2^{3}-1\right)(\operatorname{STS}(7))$ can be taken. Those blocks are $123,145,167,246,257$,
34 7, 35 6.Under that concept the program was extended to find blocks of $\operatorname{SQS}\left(2^{\mathrm{n}}-1\right)$-design.

PROGARM TO FIND STS( $\left.2^{\mathrm{n}}-1\right)$ DESIGN

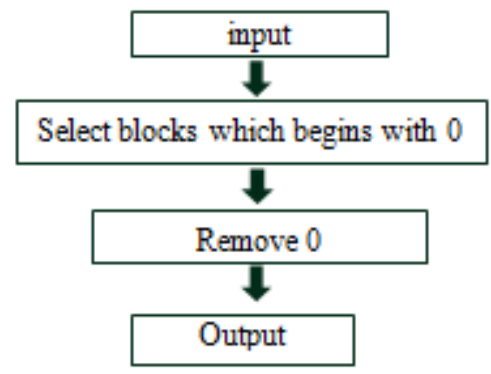

Here input was blocks of SQS $\left(2^{\mathrm{n}}\right)$-design. Then blocks which begin with 0 were selected. In those blocks 0 was removed \& obtained STS $\left(2^{\mathrm{n}}-1\right)$-design as output. After that using method of previous program $2 \&$ program 3 , incidence matrix \& complete graph were taken.

\section{Results and Discussion}

Using the program the blocks of SQS $\left(2^{\mathrm{n}}\right)$-design, number of blocks, incidence matrices, and complete graphs with $2^{\mathrm{n}}$ number of vertices were manifestly obtained. It was needed $30,60,90$ seconds to run the program for SQS(8), SQS(16), SQS(32) respectively. SQS(64) and SQS(128) were needed more than 8 hours to obtain a result. It was observed that these blocks have several properties such that triply transitive, automorphism-free (without SQS(8) ), heterogeneous for $\mathrm{n} \geq 3$, resolvable \& non-disjoint[4]. By extending the program to obtain blocks of $\operatorname{STS}\left(2^{\mathrm{n}}-1\right)$ design(i.e order $7,15,31,63,127,255 \&$ 511),number of blocks, incidence matrices, and complete graphs with $2^{\mathrm{n}}-1$ number of vertices were obtained as another result. Among these designs STS(15), STS(63) \& STS(255) are Kirkman triple systems[5]. All the designs are 3-chromatic STS while STS(31), STS(63), STS(127), STS(255) \& STS(511) are 4chromatic STS also[6]. And STS(31), STS(127) \& STS(511) are 6-rotational STS[5]. The incidence matrixes of those two types of designs are binary matrices and incidence graph of an incidence structure is a bipartite graph.

Blocks of SQS(16)

$\begin{array}{llllllllllll}0 & 1 & 2 & 3 & 1 & 4 & 8 & 13 & 3 & 6 & 9 & 12 \\ 0 & 1 & 4 & 5 & 1 & 4 & 9 & 12 & 3 & 6 & 10 & 15 \\ 0 & 1 & 6 & 7 & 1 & 4 & 10 & 15 & 3 & 6 & 11 & 14 \\ 0 & 1 & 8 & 9 & 1 & 4 & 11 & 14 & 3 & 7 & 8 & 12 \\ 0 & 1 & 10 & 11 & 1 & 5 & 8 & 12 & 3 & 7 & 9 & 13 \\ 0 & 1 & 12 & 13 & 1 & 5 & 9 & 13 & 3 & 7 & 10 & 14 \\ 0 & 1 & 14 & 15 & 1 & 5 & 10 & 14 & 3 & 7 & 11 & 15 \\ 0 & 2 & 4 & 6 & 1 & 5 & 11 & 15 & 4 & 5 & 6 & 7 \\ 0 & 2 & 5 & 7 & 1 & 6 & 8 & 15 & 4 & 5 & 8 & 9 \\ 0 & 2 & 8 & 10 & 1 & 6 & 9 & 14 & 4 & 5 & 10 & 11 \\ 0 & 2 & 9 & 11 & 1 & 6 & 10 & 13 & 4 & 5 & 12 & 13 \\ 0 & 2 & 12 & 14 & 1 & 6 & 11 & 12 & 4 & 5 & 14 & 15 \\ 0 & 2 & 13 & 15 & 1 & 7 & 8 & 14 & 4 & 6 & 8 & 10 \\ 0 & 3 & 4 & 7 & 1 & 7 & 9 & 15 & 4 & 6 & 9 & 11 \\ 0 & 3 & 5 & 6 & 1 & 7 & 10 & 12 & 4 & 6 & 12 & 14 \\ 0 & 3 & 8 & 11 & 1 & 7 & 11 & 13 & 4 & 6 & 13 & 15 \\ 0 & 3 & 9 & 10 & 2 & 3 & 4 & 5 & 4 & 7 & 8 & 11 \\ 0 & 3 & 12 & 15 & 2 & 3 & 6 & 7 & 4 & 7 & 9 & 10 \\ 0 & 3 & 13 & 14 & 2 & 3 & 8 & 9 & 4 & 7 & 12 & 15 \\ 0 & 4 & 8 & 12 & 2 & 3 & 10 & 11 & 4 & 7 & 13 & 14 \\ 0 & 4 & 9 & 13 & 2 & 3 & 12 & 13 & 5 & 6 & 8 & 11\end{array}$

Volume 4 Issue 12, December 2015 


$\begin{array}{llllllllllll}0 & 4 & 10 & 14 & 2 & 3 & 14 & 15 & 5 & 6 & 9 & 10 \\ 0 & 4 & 11 & 15 & 2 & 4 & 8 & 14 & 5 & 6 & 12 & 15 \\ 0 & 5 & 8 & 13 & 2 & 4 & 9 & 15 & 5 & 6 & 13 & 14 \\ 0 & 5 & 9 & 12 & 2 & 4 & 10 & 12 & 5 & 7 & 8 & 10 \\ 0 & 5 & 10 & 15 & 2 & 4 & 11 & 13 & 5 & 7 & 9 & 11 \\ 0 & 5 & 11 & 14 & 2 & 5 & 8 & 15 & 5 & 7 & 12 & 14 \\ 0 & 6 & 8 & 14 & 2 & 5 & 9 & 14 & 5 & 7 & 13 & 15 \\ 0 & 6 & 9 & 15 & 2 & 5 & 10 & 13 & 6 & 7 & 8 & 9 \\ 0 & 6 & 10 & 12 & 2 & 5 & 11 & 12 & 6 & 7 & 10 & 11 \\ 0 & 6 & 11 & 13 & 2 & 6 & 8 & 12 & 6 & 7 & 12 & 13 \\ 0 & 7 & 8 & 15 & 2 & 6 & 9 & 13 & 6 & 7 & 14 & 15 \\ 0 & 7 & 9 & 14 & 2 & 6 & 10 & 14 & 8 & 9 & 10 & 11 \\ 0 & 7 & 10 & 13 & 2 & 6 & 11 & 15 & 8 & 9 & 12 & 13 \\ 0 & 7 & 11 & 12 & 2 & 7 & 8 & 13 & 8 & 9 & 14 & 15 \\ 1 & 2 & 4 & 7 & 2 & 7 & 9 & 12 & 8 & 10 & 12 & 14 \\ 1 & 2 & 5 & 6 & 2 & 7 & 10 & 15 & 8 & 10 & 13 & 15 \\ 1 & 2 & 8 & 11 & 2 & 7 & 11 & 14 & 8 & 11 & 12 & 15 \\ 1 & 2 & 9 & 10 & 3 & 4 & 8 & 15 & 8 & 11 & 13 & 14 \\ 1 & 2 & 12 & 15 & 3 & 4 & 9 & 14 & 9 & 10 & 12 & 15 \\ 1 & 2 & 13 & 14 & 3 & 4 & 10 & 13 & 9 & 10 & 13 & 14 \\ 1 & 3 & 4 & 6 & 3 & 4 & 11 & 12 & 9 & 11 & 12 & 14 \\ 1 & 3 & 5 & 7 & 3 & 5 & 8 & 14 & 9 & 11 & 13 & 15 \\ 1 & 3 & 8 & 10 & 3 & 5 & 9 & 15 & 10 & 11 & 12 & 13 \\ 1 & 3 & 9 & 11 & 3 & 5 & 10 & 12 & 10 & 11 & 14 & 15 \\ 1 & 3 & 12 & 14 & 3 & 5 & 11 & 13 & 12 & 13 & 14 & 15 \\ 1 & 3 & 13 & 15 & 3 & 6 & 8 & 13 & & & & \end{array}$

Blocks of SQS(32)

\begin{tabular}{|c|c|c|c|c|c|c|c|c|c|c|c|}
\hline 0 & 4 & 17 & 21 & 3 & 7 & 17 & 21 & 8 & 10 & 17 & 19 \\
\hline 0 & 4 & 18 & 22 & 3 & 7 & 18 & 22 & 8 & 10 & 20 & 22 \\
\hline 0 & 4 & 19 & 23 & 3 & 7 & 19 & 23 & 8 & 10 & 21 & 23 \\
\hline 0 & 4 & 24 & 28 & 3 & 7 & 24 & 28 & 8 & 10 & 24 & 26 \\
\hline 0 & 4 & 25 & 29 & 3 & 7 & 25 & 29 & 8 & 10 & 25 & 27 \\
\hline 0 & 4 & 26 & 30 & 3 & 7 & 26 & 30 & 8 & 10 & 28 & 30 \\
\hline 0 & 4 & 27 & 31 & 3 & 7 & 27 & 31 & 8 & 10 & 29 & 31 \\
\hline 0 & 5 & 8 & 13 & 3 & 8 & 16 & 27 & 8 & 11 & 12 & 15 \\
\hline 0 & 5 & 9 & 12 & 3 & 8 & 17 & 26 & 8 & 11 & 13 & 14 \\
\hline 0 & 5 & 10 & 15 & 3 & 8 & 18 & 25 & 8 & 11 & 16 & 19 \\
\hline 0 & 5 & 11 & 14 & 3 & 8 & 19 & 24 & 8 & 11 & 17 & 18 \\
\hline 0 & 5 & 16 & 21 & 3 & 8 & 20 & 31 & 8 & 11 & 20 & 23 \\
\hline 0 & 5 & 17 & 20 & 3 & 8 & 21 & 30 & 8 & 11 & 21 & 22 \\
\hline 0 & 5 & 18 & 23 & 3 & 8 & 22 & 29 & 8 & 11 & 24 & 27 \\
\hline 0 & 5 & 19 & 22 & 3 & 8 & 23 & 28 & 8 & 11 & 25 & 26 \\
\hline 0 & 5 & 24 & 29 & 3 & 9 & 16 & 26 & 8 & 11 & 28 & 31 \\
\hline 0 & 5 & 25 & 28 & 3 & 9 & 17 & 27 & 8 & 11 & 29 & 30 \\
\hline 0 & 5 & 26 & 31 & 3 & 9 & 18 & 24 & 8 & 12 & 16 & 20 \\
\hline 0 & 5 & 27 & 30 & 3 & 9 & 19 & 25 & 8 & 12 & 17 & 21 \\
\hline 0 & 6 & 8 & 14 & 3 & 9 & 20 & 30 & 8 & 12 & 18 & 22 \\
\hline 0 & 6 & 9 & 15 & 3 & 9 & 21 & 31 & 8 & 12 & 19 & 23 \\
\hline 0 & 6 & 10 & 12 & 3 & 9 & 22 & 28 & 8 & 12 & 24 & 28 \\
\hline 0 & 6 & 11 & 13 & 3 & 9 & 23 & 29 & 8 & 12 & 25 & 29 \\
\hline 0 & 6 & 16 & 22 & 3 & 10 & 16 & 25 & 8 & 12 & 26 & 30 \\
\hline 0 & 6 & 17 & 23 & 3 & 10 & 17 & 24 & 8 & 12 & 27 & 31 \\
\hline 0 & 6 & 18 & 20 & 3 & 10 & 18 & 27 & 8 & 13 & 16 & 21 \\
\hline 0 & 6 & 19 & 21 & 3 & 10 & 19 & 26 & 8 & 13 & 17 & 20 \\
\hline 0 & 6 & 24 & 30 & 3 & 10 & 20 & 29 & 8 & 13 & 18 & 23 \\
\hline 0 & 6 & 25 & 31 & 3 & 10 & 21 & 28 & 8 & 13 & 19 & 22 \\
\hline 0 & 6 & 26 & 28 & 3 & 10 & 22 & 31 & 8 & 13 & 24 & 29 \\
\hline 0 & 6 & 27 & 29 & 3 & 10 & 23 & 30 & 8 & 13 & 25 & 28 \\
\hline 0 & 7 & 8 & 15 & 3 & 11 & 16 & 24 & 8 & 13 & 26 & 31 \\
\hline 0 & 7 & 9 & 14 & 3 & 11 & 17 & 25 & 8 & 13 & 27 & 30 \\
\hline 0 & 7 & 10 & 13 & 3 & 11 & 18 & 26 & 8 & 14 & 16 & 22 \\
\hline 0 & 7 & 11 & 12 & 3 & 11 & 19 & 27 & 8 & 14 & 17 & 23 \\
\hline 0 & 7 & 16 & 23 & 3 & 11 & 20 & 28 & 8 & 14 & 18 & 20 \\
\hline 0 & 7 & 17 & 22 & 3 & 11 & 21 & 29 & 8 & 14 & 19 & 21 \\
\hline 0 & 7 & 18 & 21 & 3 & 11 & 22 & 30 & 8 & 14 & 24 & 30 \\
\hline 0 & 7 & 19 & 20 & 3 & 11 & 23 & 31 & 8 & 14 & 25 & 31 \\
\hline 0 & 7 & 24 & 31 & 3 & 12 & 16 & 31 & 8 & 14 & 26 & 28 \\
\hline 0 & 7 & 25 & 30 & 3 & 12 & 17 & 30 & 8 & 14 & 27 & 29 \\
\hline 0 & 7 & 26 & 29 & 3 & 12 & 18 & 29 & 8 & 15 & 16 & 23 \\
\hline 0 & 7 & 27 & 28 & 3 & 12 & 19 & 28 & 8 & 15 & 17 & 22 \\
\hline 0 & 8 & 16 & 24 & 3 & 12 & 20 & 27 & 8 & 15 & 18 & 21 \\
\hline 0 & 8 & 17 & 25 & 3 & 12 & 21 & 26 & 8 & 15 & 19 & 20 \\
\hline 0 & 8 & 18 & 26 & 3 & 12 & 22 & 25 & 8 & 15 & 24 & 31 \\
\hline 0 & 8 & 19 & 27 & 3 & 12 & 23 & 24 & 8 & 15 & 25 & 30 \\
\hline 0 & 8 & 20 & 28 & 3 & 13 & 16 & 30 & 8 & 15 & 26 & 29 \\
\hline 0 & 8 & 21 & 29 & 3 & 13 & 17 & 31 & 8 & 15 & 27 & 28 \\
\hline 0 & 8 & 22 & 30 & 3 & 13 & 18 & 28 & 9 & 10 & 12 & 15 \\
\hline 0 & 8 & 23 & 31 & 3 & 13 & 19 & 29 & 9 & 10 & 13 & 14 \\
\hline 0 & 9 & 16 & 25 & 3 & 13 & 20 & 26 & 9 & 10 & 16 & 19 \\
\hline 0 & 9 & 17 & 24 & 3 & 13 & 21 & 27 & 9 & 10 & 17 & 18 \\
\hline 0 & 9 & 18 & 27 & 3 & 13 & 22 & 24 & 9 & 10 & 20 & 23 \\
\hline 0 & 9 & 19 & 26 & 3 & 13 & 23 & 25 & 9 & 10 & 21 & 22 \\
\hline 0 & 9 & 20 & 29 & 3 & 14 & 16 & 29 & 9 & 10 & 24 & 27 \\
\hline 0 & 9 & 21 & 28 & 3 & 14 & 17 & 28 & 9 & 10 & 25 & 26 \\
\hline 0 & 9 & 22 & 31 & 3 & 14 & 18 & 31 & 9 & 10 & 28 & 31 \\
\hline 0 & 9 & 23 & 30 & 3 & 14 & 19 & 30 & 9 & 10 & 29 & 30 \\
\hline 0 & 10 & 16 & 26 & 3 & 14 & 20 & 25 & 9 & 11 & 12 & 14 \\
\hline 0 & 10 & 17 & 27 & 3 & 14 & 21 & 24 & 9 & 11 & 13 & 15 \\
\hline 0 & 10 & 18 & 24 & 3 & 14 & 22 & 27 & 9 & 11 & 16 & 18 \\
\hline 0 & 10 & 19 & 25 & 3 & 14 & 23 & 26 & 9 & 11 & 17 & 19 \\
\hline 0 & 10 & 20 & 30 & 3 & 15 & 16 & 28 & 9 & 11 & 20 & 22 \\
\hline 0 & 10 & 21 & 31 & 3 & 15 & 17 & 29 & 9 & 11 & 21 & 23 \\
\hline 0 & 10 & 22 & 28 & 3 & 15 & 18 & 30 & 9 & 11 & 24 & 26 \\
\hline 0 & 10 & 23 & 29 & 3 & 15 & 19 & 31 & 9 & 11 & 25 & 27 \\
\hline 0 & 11 & 16 & 27 & 3 & 15 & 20 & 24 & 9 & 11 & 28 & 30 \\
\hline 0 & 11 & 17 & 26 & 3 & 15 & 21 & 25 & 9 & 11 & 29 & 31 \\
\hline 0 & 11 & 18 & 25 & 3 & 15 & 22 & 26 & 9 & 12 & 16 & 21 \\
\hline 0 & 11 & 19 & 24 & 3 & 15 & 23 & 27 & 9 & 12 & 17 & 20 \\
\hline 0 & 11 & 20 & 31 & 4 & 5 & 6 & 7 & 9 & 12 & 18 & 23 \\
\hline 0 & 11 & 21 & 30 & 4 & 5 & 8 & 9 & 9 & 12 & 19 & 22 \\
\hline 0 & 11 & 22 & 29 & 4 & 5 & 10 & 11 & 9 & 12 & 24 & 29 \\
\hline 0 & 11 & 23 & 28 & 4 & 5 & 12 & 13 & 9 & 12 & 25 & 28 \\
\hline 0 & 12 & 16 & 28 & 4 & 5 & 14 & 15 & 9 & 12 & 26 & 31 \\
\hline 0 & 12 & 17 & 29 & 4 & 5 & 16 & 17 & 9 & 12 & 27 & 30 \\
\hline 0 & 12 & 18 & 30 & 4 & 5 & 18 & 19 & 9 & 13 & 16 & 20 \\
\hline 0 & 12 & 19 & 31 & 4 & 5 & 20 & 21 & 9 & 13 & 17 & 21 \\
\hline
\end{tabular}

Volume 4 Issue 12, December 2015

$\begin{array}{lll}11 & 22 & 26\end{array}$

$\begin{array}{lll}11 & 23 & 27\end{array}$

$\begin{array}{lll}12 & 17 & 26\end{array}$

$\begin{array}{lll}12 & 18 & 25\end{array}$

$\begin{array}{lll}12 & 19 & 24\end{array}$

$12 \quad 21 \quad 30$

$\begin{array}{lll}12 & 22 & 29\end{array}$

$12 \quad 23 \quad 28$

$\begin{array}{lll}13 & 16 & 26 \\ 13 & 17 & 27\end{array}$

$13 \quad 18 \quad 24$

$\begin{array}{lll}13 & 19 & 25\end{array}$

$\begin{array}{lll}13 & 20 & 30\end{array}$

$\begin{array}{lll}13 & 21 & 31\end{array}$

$\begin{array}{lll}13 & 22 & 28\end{array}$

$\begin{array}{lll}13 & 23 & 29\end{array}$

$\begin{array}{lll}14 & 17 & 24\end{array}$

$\begin{array}{lll}14 & 18 & 27\end{array}$

$\begin{array}{lll}14 & 19 & 26\end{array}$

$\begin{array}{lll}14 & 20 & 29\end{array}$

$\begin{array}{lll}14 & 21 & 28\end{array}$

$\begin{array}{lll}14 & 22 & 31\end{array}$

$\begin{array}{lll}15 & 16 & 24\end{array}$

$\begin{array}{lll}15 & 17 & 25\end{array}$

$\begin{array}{lll}15 & 18 & 26\end{array}$

$\begin{array}{lll}15 & 19 & 27 \\ 15 & 20 & 28\end{array}$

$\begin{array}{lll}15 & 21 & 29\end{array}$

$\begin{array}{lll}15 & 22 & 30\end{array}$

$\begin{array}{lll}15 & 23 & 31\end{array}$

$\begin{array}{lll}9 & 10 & 11\end{array}$

$\begin{array}{lll}9 & 14 & 15\end{array}$

$\begin{array}{lll}9 & 18 & 19\end{array}$

$9 \quad 20 \quad 21$

$922 \quad 23$

$\begin{array}{lll}9 & 24 & 25 \\ 9 & 26 & 27\end{array}$

$\begin{array}{lll}9 & 28 & 29\end{array}$

$930 \quad 31$

$\begin{array}{lll}10 & 12 & 14\end{array}$

$\begin{array}{lll}10 & 13 & 15 \\ 10 & 16 & 18\end{array}$ 


\begin{tabular}{|c|c|c|c|c|c|c|c|c|c|c|c|c|c|c|c|c|c|c|c|}
\hline & 12 & 20 & 24 & 4 & 5 & 22 & 23 & 9 & 13 & 18 & 22 & 1 & 5 & 27 & 31 & 4 & 14 & 18 & 24 \\
\hline 0 & 12 & 21 & 25 & 4 & 5 & 24 & 25 & 9 & 13 & 19 & 23 & 1 & 6 & 8 & 15 & 4 & 14 & 19 & 25 \\
\hline 0 & 12 & 22 & 26 & 4 & 5 & 26 & 27 & 9 & 13 & 24 & 28 & 1 & 6 & 9 & 14 & 4 & 14 & 20 & 30 \\
\hline 0 & 12 & 23 & 27 & 4 & 5 & 28 & 29 & 9 & 13 & 25 & 29 & 1 & 6 & 10 & 13 & 4 & 14 & 21 & 31 \\
\hline 0 & 13 & 16 & 29 & 4 & 5 & 30 & 31 & 9 & 13 & 26 & 30 & 1 & 6 & 11 & 12 & 4 & 14 & 22 & 28 \\
\hline 0 & 13 & 17 & 28 & 4 & 6 & 8 & 10 & 9 & 13 & 27 & 31 & 1 & 6 & 16 & 23 & 4 & 14 & 23 & 29 \\
\hline 0 & 13 & 18 & 31 & 4 & 6 & 9 & 11 & 9 & 14 & 16 & 23 & 1 & 6 & 17 & 22 & 4 & 15 & 16 & 27 \\
\hline 0 & 13 & 19 & 30 & 4 & 6 & 12 & 14 & 9 & 14 & 17 & 22 & 1 & 6 & 18 & 21 & 4 & 15 & 17 & 26 \\
\hline 0 & 13 & 20 & 25 & 4 & 6 & 13 & 15 & 9 & 14 & 18 & 21 & 1 & 6 & 19 & 20 & 4 & 15 & 18 & 25 \\
\hline 0 & 13 & 21 & 24 & 4 & 6 & 16 & 18 & 9 & 14 & 19 & 20 & 1 & 6 & 24 & 31 & 4 & 15 & 19 & 24 \\
\hline 0 & 13 & 22 & 27 & 4 & 6 & 17 & 19 & 9 & 14 & 24 & 31 & 1 & 6 & 25 & 30 & 4 & 15 & 20 & 31 \\
\hline 0 & 13 & 23 & 26 & 4 & 6 & 20 & 22 & 9 & 14 & 25 & 30 & 1 & 6 & 26 & 29 & 4 & 15 & 21 & 30 \\
\hline 0 & 14 & 16 & 30 & 4 & 6 & 21 & 23 & 9 & 14 & 26 & 29 & 1 & 6 & 27 & 28 & 4 & 15 & 22 & 29 \\
\hline 0 & 14 & 17 & 31 & 4 & 6 & 24 & 26 & 9 & 14 & 27 & 28 & 1 & 7 & 8 & 14 & 4 & 15 & 23 & 28 \\
\hline 0 & 14 & 18 & 28 & 4 & 6 & 25 & 27 & 9 & 15 & 16 & 22 & 1 & 7 & 9 & 15 & 5 & 6 & 8 & 11 \\
\hline 0 & 14 & 19 & 29 & 4 & 6 & 28 & 30 & 9 & 15 & 17 & 23 & 1 & 7 & 10 & 12 & 5 & 6 & 9 & 10 \\
\hline 0 & 14 & 20 & 26 & 4 & 6 & 29 & 31 & 9 & 15 & 18 & 20 & 1 & 7 & 11 & 13 & 5 & 6 & 12 & 15 \\
\hline 0 & 14 & 21 & 27 & 4 & 7 & 8 & 11 & 9 & 15 & 19 & 21 & 1 & 7 & 16 & 22 & 5 & 6 & 13 & 14 \\
\hline 0 & 14 & 22 & 24 & 4 & 7 & 9 & 10 & 9 & 15 & 24 & 30 & 1 & 7 & 17 & 23 & 5 & 6 & 16 & 19 \\
\hline 0 & 14 & 23 & 25 & 4 & 7 & 12 & 15 & 9 & 15 & 25 & 31 & 1 & 7 & 18 & 20 & 5 & 6 & 17 & 18 \\
\hline 0 & 15 & 16 & 31 & 4 & 7 & 13 & 14 & 9 & 15 & 26 & 28 & 1 & 7 & 19 & 21 & 5 & 6 & 20 & 23 \\
\hline 0 & 15 & 17 & 30 & 4 & 7 & 16 & 19 & 9 & 15 & 27 & 29 & 1 & 7 & 24 & 30 & 5 & 6 & 21 & 22 \\
\hline 0 & 15 & 18 & 29 & 4 & 7 & 17 & 18 & 10 & 11 & 12 & 13 & 1 & 7 & 25 & 31 & 5 & 6 & 24 & 27 \\
\hline 0 & 15 & 19 & 28 & 4 & 7 & 20 & 23 & 10 & 11 & 14 & 15 & 1 & 7 & 26 & 28 & 5 & 6 & 25 & 26 \\
\hline 0 & 15 & 20 & 27 & 4 & 7 & 21 & 22 & 10 & 11 & 16 & 17 & 1 & 7 & 27 & 29 & 5 & 6 & 28 & 31 \\
\hline 0 & 15 & 21 & 26 & 4 & 7 & 24 & 27 & 10 & 11 & 18 & 19 & 1 & 8 & 16 & 25 & 5 & 6 & 29 & 30 \\
\hline 0 & 15 & 22 & 25 & 4 & 7 & 25 & 26 & 10 & 11 & 20 & 21 & 1 & 8 & 17 & 24 & 5 & 7 & 8 & 10 \\
\hline 0 & 15 & 23 & 24 & 4 & 7 & 28 & 31 & 10 & 11 & 22 & 23 & 1 & 8 & 18 & 27 & 5 & 7 & 9 & 11 \\
\hline 1 & 2 & 4 & 7 & 4 & 7 & 29 & 30 & 10 & 11 & 24 & 25 & 1 & 8 & 19 & 26 & 5 & 7 & 12 & 14 \\
\hline 1 & 2 & 5 & 6 & 4 & 8 & 16 & 28 & 10 & 11 & 26 & 27 & 1 & 8 & 20 & 29 & 5 & 7 & 13 & 15 \\
\hline 1 & 2 & 8 & 11 & 4 & 8 & 17 & 29 & 10 & 11 & 28 & 29 & 1 & 8 & 21 & 28 & 5 & 7 & 16 & 18 \\
\hline 1 & 2 & 9 & 10 & 4 & 8 & 18 & 30 & 10 & 11 & 30 & 31 & 1 & 8 & 22 & 31 & 5 & 7 & 17 & 19 \\
\hline 1 & 2 & 12 & 15 & 4 & 8 & 19 & 31 & 10 & 12 & 16 & 22 & 1 & 8 & 23 & 30 & 5 & 7 & 20 & 22 \\
\hline 1 & 2 & 13 & 14 & 4 & 8 & 20 & 24 & 10 & 12 & 17 & 23 & 1 & 9 & 16 & 24 & 5 & 7 & 21 & 23 \\
\hline 1 & 2 & 16 & 19 & 4 & 8 & 21 & 25 & 10 & 12 & 18 & 20 & 1 & 9 & 17 & 25 & 5 & 7 & 24 & 26 \\
\hline 1 & 2 & 17 & 18 & 4 & 8 & 22 & 26 & 10 & 12 & 19 & 21 & 1 & 9 & 18 & 26 & 5 & 7 & 25 & 27 \\
\hline 1 & 2 & 20 & 23 & 4 & 8 & 23 & 27 & 10 & 12 & 24 & 30 & 1 & 9 & 19 & 27 & 5 & 7 & 28 & 30 \\
\hline 1 & 2 & 21 & 22 & 4 & 9 & 16 & 29 & 10 & 12 & 25 & 31 & 1 & 9 & 20 & 28 & 5 & 7 & 29 & 31 \\
\hline 1 & 2 & 24 & 27 & 4 & 9 & 17 & 28 & 10 & 12 & 26 & 28 & 1 & 9 & 21 & 29 & 5 & 8 & 16 & 29 \\
\hline 1 & 2 & 25 & 26 & 4 & 9 & 18 & 31 & 10 & 12 & 27 & 29 & 1 & 9 & 22 & 30 & 5 & 8 & 17 & 28 \\
\hline 1 & 2 & 28 & 31 & 4 & 9 & 19 & 30 & 10 & 13 & 16 & 23 & 1 & 9 & 23 & 31 & 5 & 8 & 18 & 31 \\
\hline 1 & 2 & 29 & 30 & 4 & 9 & 20 & 25 & 10 & 13 & 17 & 22 & 1 & 10 & 16 & 27 & 5 & 8 & 19 & 30 \\
\hline 1 & 3 & 4 & 6 & 4 & 9 & 21 & 24 & 10 & 13 & 18 & 21 & 1 & 10 & 17 & 26 & 5 & 8 & 20 & 25 \\
\hline 1 & 3 & 5 & 7 & 4 & 9 & 22 & 27 & 10 & 13 & 19 & 20 & 1 & 10 & 18 & 25 & 5 & 8 & 21 & 24 \\
\hline 1 & 3 & 8 & 10 & 4 & 9 & 23 & 26 & 10 & 13 & 24 & 31 & 1 & 10 & 19 & 24 & 5 & 8 & 22 & 27 \\
\hline 1 & 3 & 9 & 11 & 4 & 10 & 16 & 30 & 10 & 13 & 25 & 30 & 1 & 10 & 20 & 31 & 5 & 8 & 23 & 26 \\
\hline 1 & 3 & 12 & 14 & 4 & 10 & 17 & 31 & 10 & 13 & 26 & 29 & 1 & 10 & 21 & 30 & 5 & 9 & 16 & 28 \\
\hline 1 & 3 & 13 & 15 & 4 & 10 & 18 & 28 & 10 & 13 & 27 & 28 & 1 & 10 & 22 & 29 & 5 & 9 & 17 & 29 \\
\hline 1 & 3 & 16 & 18 & 4 & 10 & 19 & 29 & 10 & 14 & 16 & 20 & 1 & 10 & 23 & 28 & 5 & 9 & 18 & 30 \\
\hline 1 & 3 & 17 & 19 & 4 & 10 & 20 & 26 & 10 & 14 & 17 & 21 & 1 & 11 & 16 & 26 & 5 & 9 & 19 & 31 \\
\hline 1 & 3 & 20 & 22 & 4 & 10 & 21 & 27 & 10 & 14 & 18 & 22 & 1 & 11 & 17 & 27 & 5 & 9 & 20 & 24 \\
\hline 1 & 3 & 21 & 23 & 4 & 10 & 22 & 24 & 10 & 14 & 19 & 23 & 1 & 11 & 18 & 24 & 5 & 9 & 21 & 25 \\
\hline 1 & 3 & 24 & 26 & 4 & 10 & 23 & 25 & 10 & 14 & 24 & 28 & 1 & 11 & 19 & 25 & 5 & 9 & 22 & 26 \\
\hline 1 & 3 & 25 & 27 & 4 & 11 & 16 & 31 & 10 & 14 & 25 & 29 & 1 & 11 & 20 & 30 & 5 & 9 & 23 & 27 \\
\hline 1 & 3 & 28 & 30 & 4 & 11 & 17 & 30 & 10 & 14 & 26 & 30 & 1 & 11 & 21 & 31 & 5 & 10 & 16 & 31 \\
\hline 1 & 3 & 29 & 31 & 4 & 11 & 18 & 29 & 10 & 14 & 27 & 31 & 1 & 11 & 22 & 28 & 5 & 10 & 17 & 30 \\
\hline 1 & 4 & 8 & 13 & 4 & 11 & 19 & 28 & 10 & 15 & 16 & 21 & 1 & 11 & 23 & 29 & 5 & 10 & 18 & 29 \\
\hline 1 & 4 & 9 & 12 & 4 & 11 & 20 & 27 & 10 & 15 & 17 & 20 & 1 & 12 & 16 & 29 & 5 & 10 & 19 & 28 \\
\hline 1 & 4 & 10 & 15 & 4 & 11 & 21 & 26 & 10 & 15 & 18 & 23 & 1 & 12 & 17 & 28 & 5 & 10 & 20 & 27 \\
\hline 1 & 4 & 11 & 14 & 4 & 11 & 22 & 25 & 10 & 15 & 19 & 22 & 1 & 12 & 18 & 31 & 5 & 10 & 21 & 26 \\
\hline 1 & 4 & 16 & 21 & 4 & 11 & 23 & 24 & 10 & 15 & 24 & 29 & 1 & 12 & 19 & 30 & 5 & 10 & 22 & 25 \\
\hline 1 & 4 & 17 & 20 & 4 & 12 & 16 & 24 & 10 & 15 & 25 & 28 & 1 & 12 & 20 & 25 & 5 & 10 & 23 & 24 \\
\hline 1 & 4 & 18 & 23 & 4 & 12 & 17 & 25 & 10 & 15 & 26 & 31 & 1 & 12 & 21 & 24 & 5 & 11 & 16 & 30 \\
\hline 1 & 4 & 19 & 22 & 4 & 12 & 18 & 26 & 10 & 15 & 27 & 30 & 1 & 12 & 22 & 27 & 5 & 11 & 17 & 31 \\
\hline 1 & 4 & 24 & 29 & 4 & 12 & 19 & 27 & 11 & 12 & 16 & 23 & 1 & 12 & 23 & 26 & 5 & 11 & 18 & 28 \\
\hline 1 & 4 & 25 & 28 & 4 & 12 & 20 & 28 & 11 & 12 & 17 & 22 & 1 & 13 & 16 & 28 & 5 & 11 & 19 & 29 \\
\hline 1 & 4 & 26 & 31 & 4 & 12 & 21 & 29 & 11 & 12 & 18 & 21 & 1 & 13 & 17 & 29 & 5 & 11 & 20 & 26 \\
\hline 1 & 4 & 27 & 30 & 4 & 12 & 22 & 30 & 11 & 12 & 19 & 20 & 1 & 13 & 18 & 30 & 5 & 11 & 21 & 27 \\
\hline 1 & 5 & 8 & 12 & 4 & 12 & 23 & 31 & 11 & 12 & 24 & 31 & 1 & 13 & 19 & 31 & 5 & 11 & 22 & 24 \\
\hline 1 & 5 & 9 & 13 & 4 & 13 & 16 & 25 & 11 & 12 & 25 & 30 & 1 & 13 & 20 & 24 & 5 & 11 & 23 & 25 \\
\hline 1 & 5 & 10 & 14 & 4 & 13 & 17 & 24 & 11 & 12 & 26 & 29 & 1 & 13 & 21 & 25 & 5 & 12 & 16 & 25 \\
\hline 1 & 5 & 11 & 15 & 4 & 13 & 18 & 27 & 11 & 12 & 27 & 28 & 1 & 13 & 22 & 26 & 5 & 12 & 17 & 24 \\
\hline 1 & 5 & 16 & 20 & 4 & 13 & 19 & 26 & 11 & 13 & 16 & 22 & 1 & 13 & 23 & 27 & 5 & 12 & 18 & 27 \\
\hline 1 & 5 & 17 & 21 & 4 & 13 & 20 & 29 & 11 & 13 & 17 & 23 & 1 & 14 & 16 & 31 & 5 & 12 & 19 & 26 \\
\hline 1 & 5 & 18 & 22 & 4 & 13 & 21 & 28 & 11 & 13 & 18 & 20 & 1 & 14 & 17 & 30 & 5 & 12 & 20 & 29 \\
\hline 1 & 5 & 19 & 23 & 4 & 13 & 22 & 31 & 11 & 13 & 19 & 21 & 1 & 14 & 18 & 29 & 5 & 12 & 21 & 28 \\
\hline 1 & 5 & 24 & 28 & 4 & 13 & 23 & 30 & 11 & 13 & 24 & 30 & 1 & 14 & 19 & 28 & 5 & 12 & 22 & 31 \\
\hline 1 & 5 & 25 & 29 & 4 & 14 & 16 & 26 & 11 & 13 & 25 & 31 & 1 & 14 & 20 & 27 & 5 & 12 & 23 & 30 \\
\hline & 5 & 26 & 30 & 4 & 14 & 17 & 27 & 11 & 13 & 26 & 28 & 1 & 14 & 21 & 26 & 5 & 13 & 16 & 24 \\
\hline
\end{tabular}




\begin{tabular}{|c|c|c|c|c|c|c|c|c|c|c|c|c|c|c|c|c|c|c|c|}
\hline 14 & 22 & 25 & 5 & 13 & 17 & 25 & 16 & 19 & 20 & 23 & 2 & 8 & 23 & 29 & 6 & 13 & 20 & 31 & 19 \\
\hline 14 & 23 & 24 & 5 & 13 & 18 & 26 & 16 & 19 & 21 & 22 & 2 & 9 & 16 & 27 & 6 & 13 & 21 & 30 & 19 \\
\hline 15 & 16 & 30 & 5 & 13 & 19 & 27 & 16 & 19 & 24 & 27 & 2 & 9 & 17 & 26 & 6 & 13 & 22 & 29 & 19 \\
\hline 15 & 17 & 31 & 5 & 13 & 20 & 28 & 16 & 19 & 25 & 26 & 2 & 9 & 18 & 25 & 6 & 13 & 23 & 28 & 19 \\
\hline 15 & 18 & 28 & 5 & 13 & 21 & 29 & 16 & 19 & 28 & 31 & 2 & 9 & 19 & 24 & 6 & 14 & 16 & 24 & 19 \\
\hline 15 & 19 & 29 & 5 & 13 & 22 & 30 & 16 & 19 & 29 & 30 & 2 & 9 & 20 & 31 & 6 & 14 & 17 & 25 & 19 \\
\hline 15 & 20 & 26 & 5 & 13 & 23 & 31 & 16 & 20 & 24 & 28 & 2 & 9 & 21 & 30 & 6 & 14 & 18 & 26 & 19 \\
\hline 15 & 21 & 27 & 5 & 14 & 16 & 27 & 16 & 20 & 25 & 29 & 2 & 9 & 22 & 29 & 6 & 14 & 19 & 27 & 19 \\
\hline 15 & 22 & 24 & 5 & 14 & 17 & 26 & 16 & 20 & 26 & 30 & 2 & 9 & 23 & 28 & 6 & 14 & 20 & 28 & 19 \\
\hline 15 & 23 & 25 & 5 & 14 & 18 & 25 & 16 & 20 & 27 & 31 & 2 & 10 & 16 & 24 & 6 & 14 & 21 & 29 & 20 \\
\hline 3 & 4 & 5 & 5 & 14 & 19 & 24 & 16 & 21 & 24 & 29 & 2 & 10 & 17 & 25 & 6 & 14 & 22 & 30 & 20 \\
\hline 3 & 6 & 7 & 5 & 14 & 20 & 31 & 16 & 21 & 25 & 28 & 2 & 10 & 18 & 26 & 6 & 14 & 23 & 31 & 20 \\
\hline 3 & 8 & 9 & 5 & 14 & 21 & 30 & 16 & 21 & 26 & 31 & 2 & 10 & 19 & 27 & 6 & 15 & 16 & 25 & 20 \\
\hline 3 & 10 & 11 & 5 & 14 & 22 & 29 & 16 & 21 & 27 & 30 & 2 & 10 & 20 & 28 & 6 & 15 & 17 & 24 & 20 \\
\hline 3 & 12 & 13 & 5 & 14 & 23 & 28 & 16 & 22 & 24 & 30 & 2 & 10 & 21 & 29 & 6 & 15 & 18 & 27 & 20 \\
\hline 3 & 14 & 15 & 5 & 15 & 16 & 26 & 16 & 22 & 25 & 31 & 2 & 10 & 22 & 30 & 6 & 15 & 19 & 26 & 20 \\
\hline 3 & 16 & 17 & 5 & 15 & 17 & 27 & 16 & 22 & 26 & 28 & 2 & 10 & 23 & 31 & 6 & 15 & 20 & 29 & 20 \\
\hline 3 & 18 & 19 & 5 & 15 & 18 & 24 & 16 & 22 & 27 & 29 & 2 & 11 & 16 & 25 & 6 & 15 & 21 & 28 & 20 \\
\hline 3 & 20 & 21 & 5 & 15 & 19 & 25 & 16 & 23 & 24 & 31 & 2 & 11 & 17 & 24 & 6 & 15 & 22 & 31 & 20 \\
\hline 3 & 22 & 23 & 5 & 15 & 20 & 30 & 16 & 23 & 25 & 30 & 2 & 11 & 18 & 27 & 6 & 15 & 23 & 30 & 20 \\
\hline 3 & 24 & 25 & 5 & 15 & 21 & 31 & 16 & 23 & 26 & 29 & 2 & 11 & 19 & 26 & 7 & 8 & 16 & 31 & 20 \\
\hline 3 & 26 & 27 & 5 & 15 & 22 & 28 & 16 & 23 & 27 & 28 & 2 & 11 & 20 & 29 & 7 & 8 & 17 & 30 & 20 \\
\hline 3 & 28 & 29 & 5 & 15 & 23 & 29 & 17 & 18 & 20 & 23 & 2 & 11 & 21 & 28 & 7 & 8 & 18 & 29 & 21 \\
\hline 3 & 30 & 31 & 6 & 7 & 8 & 9 & 17 & 18 & 21 & 22 & 2 & 11 & 22 & 31 & 7 & 8 & 19 & 28 & 21 \\
\hline 4 & 8 & 14 & 6 & 7 & 10 & 11 & 17 & 18 & 24 & 27 & 2 & 11 & 23 & 30 & 7 & 8 & 20 & 27 & 21 \\
\hline 4 & 9 & 15 & 6 & 7 & 12 & 13 & 17 & 18 & 25 & 26 & 2 & 12 & 16 & 30 & 7 & 8 & 21 & 26 & 21 \\
\hline 4 & 10 & 12 & 6 & 7 & 14 & 15 & 17 & 18 & 28 & 31 & 2 & 12 & 17 & 31 & 7 & 8 & 22 & 25 & 21 \\
\hline 4 & 11 & 13 & 6 & 7 & 16 & 17 & 17 & 18 & 29 & 30 & 2 & 12 & 18 & 28 & 7 & 8 & 23 & 24 & 21 \\
\hline 4 & 16 & 22 & 6 & 7 & 18 & 19 & 17 & 19 & 20 & 22 & 2 & 12 & 19 & 29 & 7 & 9 & 16 & 30 & 21 \\
\hline 4 & 17 & 23 & 6 & 7 & 20 & 21 & 17 & 19 & 21 & 23 & 2 & 12 & 20 & 26 & 7 & 9 & 17 & 31 & 21 \\
\hline 4 & 18 & 20 & 6 & 7 & 22 & 23 & 17 & 19 & 24 & 26 & 2 & 12 & 21 & 27 & 7 & 9 & 18 & 28 & 22 \\
\hline 4 & 19 & 21 & 6 & 7 & 24 & 25 & 17 & 19 & 25 & 27 & 2 & 12 & 22 & 24 & 7 & 9 & 19 & 29 & 22 \\
\hline 4 & 24 & 30 & 6 & 7 & 26 & 27 & 17 & 19 & 28 & 30 & 2 & 12 & 23 & 25 & 7 & 9 & 20 & 26 & 22 \\
\hline 4 & 25 & 31 & 6 & 7 & 28 & 29 & 17 & 19 & 29 & 31 & 2 & 13 & 16 & 31 & 7 & 9 & 21 & 27 & 22 \\
\hline 4 & 26 & 28 & 6 & 7 & 30 & 31 & 17 & 20 & 24 & 29 & 2 & 13 & 17 & 30 & 7 & 9 & 22 & 24 & 24 \\
\hline 4 & 27 & 29 & 6 & 8 & 16 & 30 & 17 & 20 & 25 & 28 & 2 & 13 & 18 & 29 & 7 & 9 & 23 & 25 & 24 \\
\hline 5 & 8 & 15 & 6 & 8 & 17 & 31 & 17 & 20 & 26 & 31 & 2 & 13 & 19 & 28 & 7 & 10 & 16 & 29 & 24 \\
\hline 5 & 9 & 14 & 6 & 8 & 18 & 28 & 17 & 20 & 27 & 30 & 2 & 13 & 20 & 27 & 7 & 10 & 17 & 28 & 24 \\
\hline 5 & 10 & 13 & 6 & 8 & 19 & 29 & 17 & 21 & 24 & 28 & 2 & 13 & 21 & 26 & 7 & 10 & 18 & 31 & 24 \\
\hline 5 & 11 & 12 & 6 & 8 & 20 & 26 & 17 & 21 & 25 & 29 & 2 & 13 & 22 & 25 & 7 & 10 & 19 & 30 & 24 \\
\hline 5 & 16 & 23 & 6 & 8 & 21 & 27 & 17 & 21 & 26 & 30 & 2 & 13 & 23 & 24 & 7 & 10 & 20 & 25 & 24 \\
\hline 5 & 17 & 22 & 6 & 8 & 22 & 24 & 17 & 21 & 27 & 31 & 2 & 14 & 16 & 28 & 7 & 10 & 21 & 24 & 25 \\
\hline 5 & 18 & 21 & 6 & 8 & 23 & 25 & 17 & 22 & 24 & 31 & 2 & 14 & 17 & 29 & 7 & 10 & 22 & 27 & 25 \\
\hline 5 & 19 & 20 & 6 & 9 & 16 & 31 & 17 & 22 & 25 & 30 & 2 & 14 & 18 & 30 & 7 & 10 & 23 & 26 & 25 \\
\hline 5 & 24 & 31 & 6 & 9 & 17 & 30 & 17 & 22 & 26 & 29 & 2 & 14 & 19 & 31 & 7 & 11 & 16 & 28 & 25 \\
\hline 5 & 25 & 30 & 6 & 9 & 18 & 29 & 17 & 22 & 27 & 28 & 2 & 14 & 20 & 24 & 7 & 11 & 17 & 29 & 26 \\
\hline 5 & 26 & 29 & 6 & 9 & 19 & 28 & 17 & 23 & 24 & 30 & 2 & 14 & 21 & 25 & 7 & 11 & 18 & 30 & 26 \\
\hline 5 & 27 & 28 & 6 & 9 & 20 & 27 & 17 & 23 & 25 & 31 & 2 & 14 & 22 & 26 & 7 & 11 & 19 & 31 & 28 \\
\hline 6 & 8 & 12 & 6 & 9 & 21 & 26 & 17 & 23 & 26 & 28 & 2 & 14 & 23 & 27 & 7 & 11 & 20 & 24 & \\
\hline 6 & 9 & 13 & 6 & 9 & 22 & 25 & 17 & 23 & 27 & 29 & 2 & 15 & 16 & 29 & 7 & 11 & 21 & 25 & \\
\hline 6 & 10 & 14 & 6 & 9 & 23 & 24 & 18 & 19 & 20 & 21 & & & & & & & & & \\
\hline 6 & 11 & 15 & 6 & 10 & 16 & 28 & 18 & 19 & 22 & 23 & & & & & & & & $\mathrm{SO}$ & ג4 \\
\hline 6 & 16 & 20 & 6 & 10 & 17 & 29 & 18 & 19 & 24 & 25 & & & & & & & & & \\
\hline 6 & 17 & 21 & 6 & 10 & 18 & 30 & 18 & 19 & 26 & 27 & & & & & & & & & 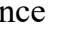 \\
\hline 6 & 18 & 22 & 6 & 10 & 19 & 31 & 18 & 19 & 28 & 29 & & cks & 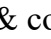 & mple & gra & h $\mathrm{x}$ & th 15 & 5 vertic & es $\mathrm{w}$ \\
\hline 6 & 19 & 23 & 6 & 10 & 20 & 24 & 18 & 19 & 30 & 31 & & & & & & & & & \\
\hline 6 & 24 & 28 & 6 & 10 & 21 & 25 & 18 & 20 & 24 & 30 & & ock & of & $\mathrm{S}(1$ & & & & & \\
\hline 6 & 25 & 29 & 6 & 10 & 22 & 26 & 18 & 20 & 25 & 31 & D & 2 & $\begin{array}{c}11 \\
3\end{array}$ & 2 & & 10 & 3 & & 14 \\
\hline 6 & 26 & 30 & 6 & 10 & 23 & 27 & 18 & 20 & 26 & 28 & 1 & $\begin{array}{l}2 \\
4\end{array}$ & $\begin{array}{l}3 \\
5\end{array}$ & $\begin{array}{l}2 \\
2\end{array}$ & $\begin{array}{l}8 \\
9\end{array}$ & $\begin{array}{l}10 \\
11\end{array}$ & $\begin{array}{l}3 \\
4\end{array}$ & $\begin{array}{l}13 \\
8\end{array}$ & $\begin{array}{l}14 \\
12\end{array}$ \\
\hline 6 & 27 & 31 & 6 & 11 & 16 & 29 & 18 & 20 & 27 & 29 & 1 & $\begin{array}{l}4 \\
6\end{array}$ & $\begin{array}{l}5 \\
7\end{array}$ & 2 & $\begin{array}{l}9 \\
12\end{array}$ & $\begin{array}{l}11 \\
14\end{array}$ & $\begin{array}{l}4 \\
4\end{array}$ & $\begin{array}{l}8 \\
9\end{array}$ & $\begin{array}{l}12 \\
13\end{array}$ \\
\hline 7 & 8 & 13 & 6 & 11 & 17 & 28 & 18 & 21 & 24 & 31 & 1 & $\begin{array}{l}6 \\
8\end{array}$ & $\begin{array}{l}7 \\
0\end{array}$ & 2 & 12 & $\begin{array}{l}14 \\
15\end{array}$ & 4 & 9 & 13 \\
\hline 7 & 9 & 12 & 6 & 11 & 18 & 31 & 18 & 21 & 25 & 30 & 1 & 8 & 9 & 2 & 13 & 15 & 4 & 10 & 14 \\
\hline 7 & 10 & 15 & 6 & 11 & 19 & 30 & 18 & 21 & 26 & 29 & 1 & 10 & 11 & 3 & 4 & 7 & 4 & 11 & 15 \\
\hline 7 & 11 & 14 & 6 & 11 & 20 & 25 & 18 & 21 & 27 & 28 & 1 & 12 & 13 & 3 & 5 & 6 & 5 & 8 & 13 \\
\hline 7 & 16 & 21 & 6 & 11 & 21 & 24 & 18 & 22 & 24 & 28 & 1 & 14 & 15 & 3 & 8 & 11 & 5 & 9 & 12 \\
\hline 7 & 17 & 20 & 6 & 11 & 22 & 27 & 18 & 22 & 25 & 29 & 2 & 4 & 6 & 3 & 9 & 10 & 5 & 10 & 15 \\
\hline 7 & 18 & 23 & 6 & 11 & 23 & 26 & 18 & 22 & 26 & 30 & 2 & 5 & 7 & 3 & 12 & 15 & 5 & 11 & 14 \\
\hline 7 & 19 & 22 & 6 & 12 & 16 & 26 & 18 & 22 & 27 & 31 & & & & & & & & & \\
\hline 7 & 24 & 29 & 6 & 12 & 17 & 27 & 18 & 23 & 24 & 29 & & ock & of $S$ & $\mathrm{TS}(3$ & & & & & \\
\hline 7 & 25 & 28 & 6 & 12 & 18 & 24 & 18 & 23 & 25 & 28 & & & & & & & & & \\
\hline 7 & 26 & 31 & 6 & 12 & 19 & 25 & 18 & 23 & 26 & 31 & 1 & 2 & 3 & 3 & 24 & 27 & 6 & 27 & 29 \\
\hline 7 & 27 & 30 & 6 & 12 & 20 & 30 & 18 & 23 & 27 & 30 & 1 & 4 & 5 & 3 & 25 & 26 & 7 & 8 & 15 \\
\hline 8 & 16 & 26 & 6 & 12 & 21 & 31 & 19 & 20 & 24 & 31 & 1 & 6 & 7 & 3 & 28 & 31 & 7 & 9 & 14 \\
\hline 8 & 17 & 27 & 6 & 12 & 22 & 28 & 19 & 20 & 25 & 30 & 1 & 8 & 9 & 3 & 29 & 30 & 7 & 10 & 13 \\
\hline 8 & 18 & 24 & 6 & 12 & 23 & 29 & 19 & 20 & 26 & 29 & 1 & 10 & 11 & 4 & 8 & 12 & 7 & 11 & 12 \\
\hline 8 & 19 & 25 & 6 & 13 & 16 & 27 & 19 & 20 & 27 & 28 & 1 & 12 & 13 & 4 & 9 & 13 & 7 & 16 & 23 \\
\hline 8 & 20 & 30 & 6 & 13 & 17 & 26 & 19 & 21 & 24 & 30 & 1 & 14 & 15 & 4 & 10 & 14 & 7 & 17 & 22 \\
\hline 8 & 21 & 31 & 6 & 13 & 18 & 25 & 19 & 21 & 25 & 31 & 1 & 16 & 17 & 4 & 11 & 15 & 7 & 18 & 21 \\
\hline 8 & 22 & 28 & 6 & 13 & 19 & 24 & 19 & 21 & 26 & 28 & 1 & 18 & 19 & 4 & 16 & 20 & 7 & 19 & 20 \\
\hline
\end{tabular}

\section{Volume 4 Issue 12, December 2015}




\section{International Journal of Science and Research (IJSR) \\ ISSN (Online): 2319-7064}

Index Copernicus Value (2013): 6.14 | Impact Factor (2014): 5.611

$\begin{array}{llllllllllll}1 & 20 & 21 & 4 & 17 & 21 & 7 & 24 & 31 & 12 & 19 & 31 \\ 1 & 22 & 23 & 4 & 18 & 22 & 7 & 25 & 30 & 12 & 20 & 24 \\ 1 & 24 & 25 & 4 & 19 & 23 & 7 & 26 & 29 & 12 & 21 & 25 \\ 1 & 26 & 27 & 4 & 24 & 28 & 7 & 27 & 28 & 12 & 22 & 26 \\ 1 & 28 & 29 & 4 & 25 & 29 & 8 & 16 & 24 & 12 & 23 & 27 \\ 1 & 30 & 31 & 4 & 26 & 30 & 8 & 17 & 25 & 13 & 16 & 29 \\ 2 & 4 & 6 & 4 & 27 & 31 & 8 & 18 & 26 & 13 & 17 & 28 \\ 2 & 5 & 7 & 5 & 8 & 13 & 8 & 19 & 27 & 13 & 18 & 31 \\ 2 & 8 & 10 & 5 & 9 & 12 & 8 & 20 & 28 & 13 & 19 & 30 \\ 2 & 9 & 11 & 5 & 10 & 15 & 8 & 21 & 29 & 13 & 20 & 25 \\ 2 & 12 & 14 & 5 & 11 & 14 & 8 & 22 & 30 & 13 & 21 & 24 \\ 2 & 13 & 15 & 5 & 16 & 21 & 8 & 23 & 31 & 13 & 22 & 27 \\ 2 & 16 & 18 & 5 & 17 & 20 & 9 & 16 & 25 & 13 & 23 & 26 \\ 2 & 17 & 19 & 5 & 18 & 23 & 9 & 17 & 24 & 14 & 16 & 30 \\ 2 & 20 & 22 & 5 & 19 & 22 & 9 & 18 & 27 & 14 & 17 & 31 \\ 2 & 21 & 23 & 5 & 24 & 29 & 9 & 19 & 26 & 14 & 18 & 28 \\ 2 & 24 & 26 & 5 & 25 & 28 & 9 & 20 & 29 & 14 & 19 & 29 \\ 2 & 25 & 27 & 5 & 26 & 31 & 9 & 21 & 28 & 14 & 20 & 26 \\ 2 & 28 & 30 & 5 & 27 & 30 & 9 & 22 & 31 & 14 & 21 & 27 \\ 2 & 29 & 31 & 6 & 8 & 14 & 9 & 23 & 30 & 14 & 22 & 24 \\ 3 & 4 & 7 & 6 & 9 & 15 & 10 & 16 & 26 & 14 & 23 & 25 \\ 3 & 5 & 6 & 6 & 10 & 12 & 10 & 17 & 27 & 15 & 16 & 31 \\ 3 & 8 & 11 & 6 & 11 & 13 & 10 & 18 & 24 & 15 & 17 & 30 \\ 3 & 9 & 10 & 6 & 16 & 22 & 10 & 19 & 25 & 15 & 18 & 29 \\ 3 & 12 & 15 & 6 & 17 & 23 & 10 & 20 & 30 & 15 & 19 & 28 \\ 3 & 13 & 14 & 6 & 18 & 20 & 10 & 21 & 31 & 15 & 20 & 27 \\ 3 & 16 & 19 & 6 & 19 & 21 & 10 & 22 & 28 & 15 & 21 & 26 \\ 3 & 17 & 18 & 6 & 24 & 30 & 10 & 23 & 29 & 15 & 22 & 25 \\ 3 & 20 & 23 & 6 & 25 & 31 & 11 & 16 & 27 & 15 & 23 & 24 \\ 3 & 21 & 22 & 6 & 26 & 28 & 11 & 17 & 26 & & & \end{array}$

\section{Conclusions}

There are substantial application of SQS and STS in fields of communication, cryptography, and networking. Optical orthogonal codes, authentication codes, multidrop networks, software testing, group testing and super imposed codes are some of areas from that fields where SQS and STS are applied.

\section{References}

[1] Anderson, I., \& Honkala, L. (1997). A short course in combinatorial designs. 28.

[2] Haim, H., Beer, S., \& Ryser, H. J. (1972). On resolvable balanced incomplete block designs.

[3] Hartman, A. (1987). The existence of resolvable Steiner Quadruple Systems. Journal of Combinatorial Theory, 193.

[4] Linder, C. C., \& Rosa, A. (1977). Steiner Quadruple System - A survey. 148. 